\title{
Flat band ferromagnetism without connectivity conditions in the flat band
}

\author{
Miklos Gulácsi, György Kovács, and Zsolt Gulácsi \\ Department of Theoretical Physics, University of Debrecen, H-4010 Debrecen, Hungary
}

(Dated: August 13, 2014)

\begin{abstract}
It is known that a system which exhibits a half filled lowest flat band and the localized oneparticle Wannier states on the flat band satisfy the connectivity conditions, is always ferromagnetic. Without the connectivity conditions on the flat band, the system is non-magnetic. We show that this is not always true. The reason is connected to a peculiar behavior of the band situated just above the flat band.

PACS numbers: 71.10.Fd, 71.27.+a, 03.65.Aa
\end{abstract}


Flat bands represent a real driving force nowadays since they appear in a broad class of subjects of large interest, as quantum Hall effect [1], spin-quantum Hall effect [2], topological phases [2, 3], bose condensations [4], highly frustrated systems [5], delocalization effects [6] or symmetry broken ordered phases [7]. Among the ordered phases connected to flat bands, the flat band ferromagnetism [7, 8] is the most important, providing a leading mechanism especially in organic or frustrated materials - for the emergence of ferromagnetism in conditions in which magnetic atoms are completely missing from the system. In the mechanism of flat band ferromagnetism (on the lowest half filled bare flat band), it is known that the system defined on a lattice (or graph) which can be described by a Hubbard type of model, is ferromagnetic for any arbitrary small on-site Coulomb repulsion $U>0$, if and only if the corresponding one-particle localized Wannier states are in contact with each other, i.e., the connectivity condition is satisfied for the bare flat band. If however, the connectivity condition for the localized one-particle states on the flat band is not satisfied, hence the spins of the individual electrons localized on the bare flat band are unable to correlate, the system will remain paramagnetic.

Several extensions of the original flat band ferromagnetism mechanism have been worked out. For example, ferromagnetism in the vicinity of flat bands [9], or due to non-lowest energy bare flat bands [10], or on effective flat bands created by interaction in conditions in which bare flat bands are not present [11-13] and even in cases when large number of non-interacting sites are present in the system [14].

In this Letter we revisit the flat band ferromagnetism phenomenon and demonstrate rigorously, that even if the connectivity conditions are not satisfied for the one-particle localized Wannier states on the bare lowest flat band, ferromagnetism is able to appear in the system. This is caused by an often possible peculiar behavior of the dispersive band situated just above the lowest flat band, which enforces the connectivity as will be detailed below.

The technique we apply is based on positive semidefinite operator properties which allowed us to work out exact results in systems and models where exact results were unheard of before, such as: periodic Anderson model in one [15], two [16], or three [17] dimensions; disordered and interacting systems in two dimensions [18]; emergence of stripes and droplets in 2D [19]; delocalization effect caused by the on-site Coulomb interaction in 2D [6]; exact results of non-integrable quadrilateral [10] or pentagon [11, 12] chains. 
The best way to explain this new effect is through the concrete example of pentagon chains. We are studying pentagon chains because they are the building blocks of a wide class of conducting polymers [20] and are a fascinating class of materials with a wide range of applications [21]. These pentagon chain polymers have been explored and utilized intensively in the past [22]. In particular, polythiophene was studied in the search for plastic ferromagnets and, more generally, for ferromagnetism in systems made entirely of nonmagnetic elements. Just recently [11, 14] we proved that ferromagnetism does exist in these class of polymers with the use of the positive semidefinite operators.

Hereafter, using the same technique, we study another class of pentagon chain polymers, namely the poly(3-alkylthiophene) [23] which has not been studied before at all. The pentagon chain is formed by blocks (see, Fig. 1) described by a Hubbard model containing on each sites the on-site Coulomb repulsion $U>0$. The non-interacting part of the Hamiltonian is:

$$
\begin{aligned}
\hat{H}_{0} & =\sum_{\sigma} \sum_{\mathbf{i}=1}^{N_{c}}\left\{\left[t_{c} \hat{c}_{\mathbf{i}+\mathbf{r}_{6}, \sigma}^{\dagger} \hat{c}_{\mathbf{i}+\mathbf{a}, \sigma}+t_{n} \hat{c}_{\mathbf{i}+\mathbf{r}_{4}, \sigma}^{\dagger} \hat{c}_{\mathbf{i}+\mathbf{r}_{5}, \sigma}\right.\right. \\
& +t_{1}\left(\hat{c}_{\mathbf{i}+\mathbf{r}_{2}, \sigma}^{\dagger} \hat{c}_{\mathbf{i}+\mathbf{r}_{4}, \sigma}+\hat{c}_{\mathbf{i}+\mathbf{r}_{2}, \sigma}^{\dagger} \hat{c}_{\mathbf{i}+\mathbf{r}_{5}, \sigma}\right)+t\left(\hat{c}_{\mathbf{i}+\mathbf{r}_{2}, \sigma}^{\dagger} \hat{c}_{\mathbf{i}, \sigma}\right. \\
& \left.\left.+\hat{c}_{\mathbf{i}+\mathbf{r}_{6}, \sigma} \hat{c}_{\mathbf{i}+\mathbf{r}_{2}, \sigma}+\hat{c}_{\mathbf{i}, \sigma}^{\dagger} \hat{c}_{\mathbf{i}+\mathbf{r}_{4}, \sigma}+\hat{c}_{\mathbf{i}+\mathbf{r}_{5}, \sigma}^{\dagger} \hat{c}_{\mathbf{i}+\mathbf{r}_{6}, \sigma}\right)+H . c .\right] \\
& +\epsilon_{0} \hat{n}_{\mathbf{i}+\mathbf{r}_{2}, \sigma}+\epsilon_{1}\left(\hat{n}_{\mathbf{i}+\mathbf{r}_{4}, \sigma}+\hat{n}_{\mathbf{i}+\mathbf{r}_{5}, \sigma}\right) \\
& \left.+\epsilon_{2}\left(\hat{n}_{\mathbf{i}, \sigma}+\hat{n}_{\mathbf{i}+\mathbf{r}_{6}, \sigma}\right)\right\},
\end{aligned}
$$

where $N_{c}$ represents the number of cells. The sites inside the unit cell constructed at the lattice site $\mathbf{i}$ are placed at $\mathbf{i}+\mathbf{r}_{n}$, where $n=2,3, \ldots 6$ represents the in-cell notation of sites,

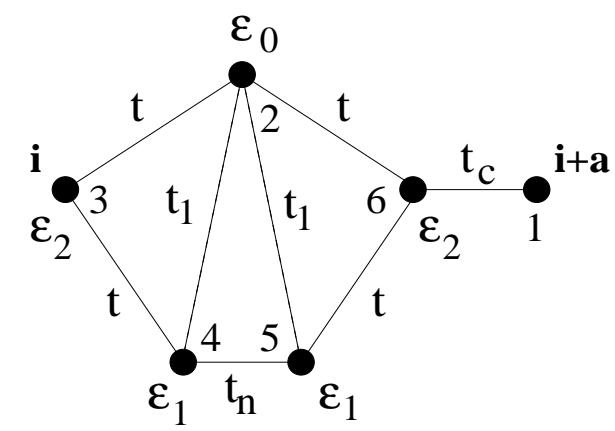

FIG. 1: The unit cell defined at the lattice site $\mathbf{i}$ of the pentagon chain under consideration. The numbers are representing the in-cell notation of sites, $t, t_{1}, \epsilon_{m}, \mathrm{~m}=0,1,2$ are the hopping matrix elements and the on-site one particle potentials present in $\hat{H}_{0}$, while $\mathbf{a}$ is the Bravais vector. 
the $n=1$ value denotes the $\mathbf{i}+\mathbf{a}$ site where $\mathbf{a}$ is the Bravais vector, and for mathematical convenience $\mathbf{r}_{3}=0$ is considered. The one-particle on-site potentials are denoted by $\epsilon_{0}$ on the site $\mathbf{i}+\mathbf{r}_{2} ; \epsilon_{1}$ on sites $\mathbf{i}+\mathbf{r}_{4} \mathbf{i}+\mathbf{r}_{5} ;$ while on the sites $\mathbf{i}$ and $\mathbf{i}+\mathbf{r}_{6}$ (hence also on $\mathbf{i}+\mathbf{a}$ in the next cell) by $\epsilon_{2}$. The nearest neighbor hopping matrix elements are $t_{4,5}=t_{n}$ on the lower horizontal bond of the cell; $t_{6,1}=t_{c}$ on the horizontal external connecting bond of the cell, and $t_{3,4}=t_{5,6}=t_{2,3}=t_{6,2}=t$ on the circumference of the pentagon. In the poly(3-alkylthiophene) polymers there is also a next-nearest neighbor hopping, denoted by $t_{1}$, because at the site $n=2$ there is always a bigger atom present relative to sites $n=3,4,5,6$. Note that we have $m=5$ sites per unit cell, hence 5 sub-lattices are present providing 5 bands in the system.

The full Hamiltonian is $\hat{H}=\hat{H}_{0}+\hat{H}_{U}$, where the interacting part is given by $\hat{H}_{U}=$ $\sum_{\mathbf{i}=1}^{N_{c}} \sum_{n=2}^{6} U_{\mathbf{i}+\mathbf{r}_{n}} \hat{n}_{\mathbf{i}+\mathbf{r}_{n}, \uparrow} \hat{n}_{\mathbf{i}+\mathbf{r}_{n}, \downarrow}, U_{\mathbf{j}}=U>0$ for all $\mathbf{j}$.

In order to find the band structure of $\hat{H}_{0}$ we Fourier transform the Fermi operators from the Hamiltonian via $\hat{c}_{\mathbf{i}+\mathbf{r}_{n}, \sigma}=\left(1 / \sqrt{N_{c}}\right) \sum_{\mathbf{k}=1}^{N_{c}} e^{-i \mathbf{k i}} e^{-i \mathbf{k r}_{n}} \hat{c}_{n, \mathbf{k}, \sigma}$, where $\mathbf{k}$ is directed along the line of the chain, and one has $|\mathbf{k}|=k=2 m \pi /\left(a N_{c}\right), m=0,1,2, \ldots, N_{c}-1,|\mathbf{a}|=a$ being the lattice constant. After this step, the non-interacting part of the Hamiltonian becomes

$$
\hat{H}_{0}=\sum_{\sigma} \sum_{k=1}^{N_{c}}\left(\hat{c}_{2, k, \sigma}^{\dagger}, \hat{c}_{3, k, \sigma}^{\dagger}, \ldots, \hat{c}_{6, k, \sigma}^{\dagger}\right) \tilde{M}\left(\begin{array}{c}
\hat{c}_{2, k, \sigma} \\
\hat{c}_{3, k, \sigma} \\
\ldots . \\
\hat{c}_{6, k, \sigma}
\end{array}\right)
$$

where the $5 \times 5$ matrix $\tilde{M}$ is:

$$
\tilde{M}=\left(\begin{array}{ccccc}
\epsilon_{0} & t e^{+i \frac{k b}{2}} & t_{1} e^{i k\left(\frac{b}{2}-b_{2}\right)} & t_{1} e^{-i k\left(\frac{b}{2}-b_{2}\right)} & t e^{-i \frac{k b}{2}} \\
t e^{-i \frac{k b}{2}} & \epsilon_{2} & t e^{-i k b_{2}} & 0 & t_{c} e^{i k b^{\prime}} \\
t_{1} e^{-i k\left(\frac{b}{2}-b_{2}\right)} & t e^{+i k b_{2}} & \epsilon_{1} & t_{n} e^{-i k b_{1}} & 0 \\
t_{1} e^{i k\left(\frac{b}{2}-b_{2}\right)} & 0 & t_{n} e^{+i k b_{1}} & \epsilon_{1} & t e^{-i k b_{2}} \\
t e^{+i \frac{k b}{2}} & t_{c} e^{-i k b^{\prime}} & 0 & t^{\prime \prime} e^{+i k b_{2}} & \epsilon_{2}
\end{array}\right)
$$

Here distances $b_{\alpha}$ are expressed by the unit vector $\mathbf{u}$ directed along $\mathbf{k}$, obtaining $b_{1}=$ $\left|\mathbf{r}_{5}-\mathbf{r}_{4}\right|, b_{2}=\left|\left(\mathbf{r}_{4}-\mathbf{r}_{3}\right) \mathbf{u}\right|, b^{\prime}=\left|\mathbf{a}-\mathbf{r}_{6}\right|, b=\left|\mathbf{r}_{6}-\mathbf{r}_{3}\right|, a=b+b^{\prime}$, and $b=b_{1}+2 b_{2}$. The band structure in obtained by diagonalizing $\tilde{M}$. 
This yields $E_{\mathbf{k}}=\epsilon$ from the equation $0=A+B \cos (a k)$, where

$$
\begin{aligned}
A & =\left[\left(\epsilon_{0}-\epsilon\right)\left(\epsilon_{2}-\epsilon\right)-2 t^{2}\right] \\
& \times\left[\left(\epsilon_{1}-\epsilon\right)^{2}\left(\epsilon_{2}-\epsilon\right)-t^{2}\left(\epsilon_{1}-\epsilon\right)-t_{n}^{2}\left(\epsilon_{2}-\epsilon\right)\right] \\
& +2\left[t^{2}-t_{1}\left(\epsilon_{2}-\epsilon\right)\right] \\
& \times\left[\left(\epsilon_{1}-\epsilon\right)\left(\epsilon_{2}-\epsilon\right) t_{1}+t_{n} t^{2}-t_{1} t^{2}-\left(\epsilon_{2}-\epsilon\right) t_{1} t_{n}\right] \\
& +t t_{1}\left\{t\left[\left(\epsilon_{1}-\epsilon\right)\left(\epsilon_{2}-\epsilon\right)-t^{2}\right]\right. \\
& \left.+t\left[\left(\epsilon_{1}-\epsilon\right)\left(\epsilon_{2}-\epsilon\right)-t^{2}\right]\right\} \\
& -t^{2}\left(\epsilon_{0}-\epsilon\right)\left[\left(\epsilon_{1}-\epsilon\right)\left(\epsilon_{2}-\epsilon\right)-t^{2}\right] \\
& +2 t_{1}^{2} t_{c}^{2}\left[\left(\epsilon_{1}-\epsilon\right)-t_{n}\right]-\left(\epsilon_{0}-\epsilon\right) t_{c}^{2}\left[\left(\epsilon_{1}-\epsilon\right)^{2}-t_{n}^{2}\right],
\end{aligned}
$$

and

$$
\begin{aligned}
B & =2\left\{t^{2} t_{c}\left[\left(\epsilon_{1}-\epsilon\right)^{2}-t_{n}^{2}\right]+2 t t_{c} t_{1}\left[t_{n} t-t\left(\epsilon_{1}-\epsilon\right)\right]\right. \\
& \left.-t^{2} t_{c}\left[t_{n}\left(\epsilon_{0}-\epsilon\right)-t_{1}^{2}\right]\right\} .
\end{aligned}
$$

From this the flat band condition is obtained when simultaneously $A=0$ and $B=0$ :

$$
\begin{aligned}
\epsilon_{0} & =2\left(\epsilon_{1}-t_{n}\right)+\frac{\left(t_{1}-\epsilon_{1}+t_{n}\right)^{2}}{t_{n}} \\
\epsilon_{2} & =\frac{t^{2}}{\epsilon_{1}-t_{n}}+\frac{t_{c}^{2}\left(\epsilon_{1}-t_{n}\right)}{\epsilon_{2}\left(\epsilon_{1}-t_{n}\right)-t^{2}}
\end{aligned}
$$

In order to place the flat band in lowest position, supplementary conditions must be imposed, which can written as

$$
\begin{aligned}
& \epsilon_{0}, \epsilon_{1}, \epsilon_{2}>0, \quad t_{n}>0, \\
& \epsilon_{1}-t_{n}>0, \quad \epsilon_{2}\left(\epsilon_{1}-t_{n}\right)-t^{2}>0 .
\end{aligned}
$$

The ground state on the lowest flat band can be easily constructed by transforming the starting Hamiltonian in positive semidefinite form. In the case of $m=5$ sub-lattices, the use of $m-1=4$ block operators for this transformation always lead to transformation conditions which provide a flat band [13]. In the present case the used four block operators as linear combinations of fermionic operators acting on the sites of the block, are defined for 
each unit cell on three triangles and a bond as follows

$$
\begin{aligned}
& \hat{A}_{1, \mathbf{i}, \sigma}=a_{1,2} \hat{c}_{\mathbf{i}+\mathbf{r}_{2}, \sigma}+a_{1,3} \hat{c}_{\mathbf{i}+\mathbf{r}_{3}, \sigma}+a_{1,4} \hat{c}_{\mathbf{i}+\mathbf{r}_{4}, \sigma}, \\
& \hat{A}_{2, \mathbf{i}, \sigma}=a_{2,2} \hat{c}_{\mathbf{i}+\mathbf{r}_{2}, \sigma}+a_{2,4} \hat{c}_{\mathbf{i}+\mathbf{r}_{4}, \sigma}+a_{2,5} \hat{c}_{\mathbf{i}+\mathbf{r}_{5}, \sigma}, \\
& \hat{A}_{3, \mathbf{i}, \sigma}=a_{3,2} \hat{c}_{\mathbf{i}+\mathbf{r}_{2, \sigma}}+a_{3,5} \hat{c}_{\mathbf{i}+\mathbf{r}_{5}, \sigma}+a_{3,6} \hat{c}_{\mathbf{i}+\mathbf{r}_{6}, \sigma}, \\
& \hat{A}_{4, \mathbf{i}, \sigma}=a_{4,6} \hat{c}_{\mathbf{i}+\mathbf{r}_{6, \sigma}}+a_{4,1} \hat{c}_{\mathbf{i}+\mathbf{a}, \sigma},
\end{aligned}
$$

where the coefficients $a_{i, j}$ denote the numerical prefactor of the Fermi operator from the block operator $i$ at the site $\mathbf{r}_{j}$. The transformation in positive semidefinite form leads to

$$
\hat{H}=\hat{H}_{A}+\hat{H}_{U}, \quad \hat{H}_{A}=\sum_{\sigma} \sum_{\mathbf{i}=1}^{N_{c}} \sum_{\alpha=1}^{4} \hat{A}_{\alpha, \mathbf{i}, \sigma}^{\dagger} \hat{A}_{\alpha, \mathbf{i}, \sigma} .
$$

The matching equations are (note that periodic boundary conditions are used):

$$
\begin{aligned}
& t_{n}=a_{2,4}^{*} a_{2,5}, \quad t_{c}=a_{4,6}^{*} a_{4,1}, \\
& t=a_{1,2}^{*} a_{1,3}=a_{3,6}^{*} a_{3,2}=a_{1,3}^{*} a_{1,4}=a_{3,5}^{*} a_{3,6}, \\
& t_{1}=a_{2,2}^{*} a_{2,5}+a_{3,2}^{*} a_{3,5}=a_{2,2}^{*} a_{2,4}+a_{1,2}^{*} a_{1,4}, \\
& \epsilon_{0}=\left|a_{1,2}\right|^{2}+\left|a_{3,2}\right|^{2}+\left|a_{2,2}\right|^{2}, \\
& \epsilon_{1}=\left|a_{1,4}\right|^{2}+\left|a_{2,4}\right|^{2}=\left|a_{2,5}\right|^{2}+\left|a_{3,5}\right|^{2}, \\
& \epsilon_{2}=\left|a_{1,3}\right|^{2}+\left|a_{4,1}\right|^{2}=\left|a_{3,6}\right|^{2}+\left|a_{4,6}\right|^{2} .
\end{aligned}
$$

The equations (9) lead to the solution

$$
\begin{aligned}
& a_{1,2}=a_{1,4}=a_{3,2}=a_{3,5}=\operatorname{sign}(t) \sqrt{\epsilon_{1}-t_{n}} e^{i \phi_{1}}, \\
& a_{1,3}=a_{3,6}=\frac{|t|}{\sqrt{\epsilon_{1}-t_{n}}} e^{i \phi_{1}}, \\
& a_{2,4}=a_{2,5}=\sqrt{t_{n}} e^{i \phi_{2}}, \quad a_{2,2}=\frac{t_{1}-\epsilon_{1}+t_{n}}{\sqrt{t_{n}}} e^{i \phi_{2}}, \\
& a_{4,1}=\sqrt{\frac{\epsilon_{2}\left(\epsilon_{1}-t_{n}\right)-t^{2}}{\epsilon_{1}-t_{n}}} e^{i \phi_{3}}, \\
& a_{4,6}=t_{c} \sqrt{\frac{\epsilon_{1}-t_{n}}{\epsilon_{2}\left(\epsilon_{1}-t_{n}\right)-t^{2}}} e^{i \phi_{3}},
\end{aligned}
$$

where $\phi_{\alpha}, \alpha=1,2,3$ are arbitrary phases. The conditions under which (9) has solutions, and that the obtained flat band is in the lowest position coincide to the conditions in (56). 
Once the block operators from (7) are worked out, the ground state can be easily constructed from the new block operators $\hat{B}_{\alpha_{\mathbf{i}}, \sigma_{\mathbf{i}}}^{\dagger}$ defined on all lattice sites $\mathbf{i}$ as

$$
\hat{B}_{\alpha_{\mathbf{i}}, \sigma_{\mathbf{i}}}^{\dagger}=\sum_{\mathbf{j}=1}^{N_{c}} \sum_{n=2}^{6} x_{\mathbf{j}, n} \hat{c}_{\mathbf{j}+\mathbf{r}_{n}, \sigma_{\mathbf{i}}}^{\dagger} .
$$

Here, $\alpha_{\mathbf{i}}$ is an index denoting linearly independent $\hat{B}^{\dagger}$ operators. The requirement for the $\hat{B}_{\alpha_{\mathbf{i}}, \sigma_{\mathbf{i}}}^{\dagger}$ operators is: i) to satisfy for all values of all indices the anti-commutation relations

$$
\left\{\hat{A}_{n, \mathbf{i}^{\prime}, \sigma^{\prime}}, \hat{B}_{\alpha_{\mathbf{i}}, \sigma_{\mathbf{i}}}^{\dagger}\right\}=0
$$

and ii) the product $\prod_{\mathbf{i}} \hat{B}_{\alpha_{\mathbf{i}}, \sigma_{\mathbf{i}}}^{\dagger}|0\rangle$, where $|0\rangle$ is the bare vacuum, must not introduce double occupancy in the system. In this case, at half filling lowest flat band the ground state becomes

$$
\left|\Psi_{g}\right\rangle=\prod_{\mathbf{i}=1}^{N_{c}} \hat{B}_{\alpha_{\mathbf{i}}, \sigma_{\mathbf{i}}}^{\dagger}|0\rangle .
$$

This indeed satisfies property i) since the relation $\hat{H}_{A}\left|\Psi_{g}\right\rangle=0$ is satisfied, and due to the property ii), also $\hat{H}_{U}\left|\Psi_{g}\right\rangle=0$ holds. The uniqueness proof can be easily done, for example, on the line of [12].

On the bare flat band the one-particle states are given by $\left|\phi_{\mathbf{i}, \sigma_{\mathbf{i}}}\right\rangle=\hat{B}_{\alpha_{\mathbf{i}}, \sigma_{\mathbf{i}}}^{\dagger}|0\rangle$. Usually these are localized states because the solutions of (12) provide $\hat{B}_{\alpha_{\mathbf{i}}, \sigma_{\mathbf{i}}}^{\dagger}$ operators which act only on the sites of a finite block.

The connectivity conditions exist if the neighboring $\hat{B}_{\alpha_{\mathbf{i}}, \sigma_{\mathbf{i}}}^{\dagger}$ operators are in contact with each other at least on one site. If this property however is missing, the localized one-particle states on the flat band are called "disconnected", i.e., do not satisfying the connectivity condition.

There is a great difference between the physical properties of the ground state (13) with connectivity, or without connectivity conditions. This is because when connectivity exists, the $\hat{B}_{\alpha_{\mathbf{i}}, \sigma_{\mathbf{i}}}^{\dagger}$ operators are in contact with each other. Thus, in order to not have double occupancy (condition ii) under (11)), i.e., to avoid the increase in energy caused by the Hubbard interaction, the system must fix the spin index of all operators in (13). Consequently the system becomes ferromagnetic. This is the flat band ferromagnetism phase. Contrary to this, when the connectivity condition does not exist and the different $\hat{B}_{\alpha_{\mathbf{i}}, \sigma_{\mathbf{i}}}^{\dagger}$ operators are not in contact with each other, the spin on individual $\hat{B}_{\alpha_{\mathbf{i}}, \sigma_{\mathbf{i}}}^{\dagger}$ operators can remain arbitrary. This 
is due to the fact that, double occupancy does not occur and hence the Hubbard interaction is completely avoided. In this case the $\left|\Psi_{g}\right\rangle$ ground state from (13) becomes paramagnetic.

In the present case non-connected $\hat{B}_{\alpha_{\mathbf{i}}, \sigma_{\mathbf{i}}}^{\dagger}$ block operators can appear as defined on internal triangle blocks in each cell, as depicted in Fig.2. If such a type of solution of (12) exists, it must exist for all $\mathbf{j}$ lattice sites

$$
\begin{aligned}
& x_{2}=x_{\mathbf{j}, 2} \neq 0, \quad x_{4}=x_{\mathbf{j}, 4} \neq 0, \\
& x_{5}=x_{\mathbf{j}, 5} \neq 0, \quad x_{\mathbf{j}, n=3,6}=0 .
\end{aligned}
$$

In this case the $\hat{B}_{\alpha_{\mathbf{i}}, \sigma_{\mathbf{i}}}^{\dagger}$ operators become

$$
\begin{aligned}
\hat{B}_{\mathbf{i}, \sigma_{\mathbf{i}}}^{\dagger} & =\hat{B}_{\alpha_{\mathbf{i}}, \sigma_{\mathbf{i}}}^{\dagger} \\
& =x_{2} \hat{c}_{\mathbf{i}+\mathbf{r}_{2}, \sigma_{\mathbf{i}}}^{\dagger}+x_{4} \hat{c}_{\mathbf{i}+\mathbf{r}_{4}, \sigma_{\mathbf{i}}}^{\dagger}+x_{2} \hat{C}_{\mathbf{i}+\mathbf{r}_{2}, \sigma_{\mathbf{i}}}^{\dagger} .
\end{aligned}
$$

In order to satisfy (14, 15), the equation (12) gives the system of equations

$$
\begin{aligned}
& a_{1,2} x_{2}+a_{1,4} x_{4}=0, \\
& a_{3,2} x_{2}+a_{3,5} x_{5}=0, \\
& a_{2,2} x_{2}+a_{2,4} x_{4}+a_{2,5} x_{5}=0,
\end{aligned}
$$

which provides $x_{2}, x_{4}, x_{5} \neq 0$ nontrivial solution only if

$$
t_{1}=\epsilon_{1}+\left|t_{n}\right|
$$

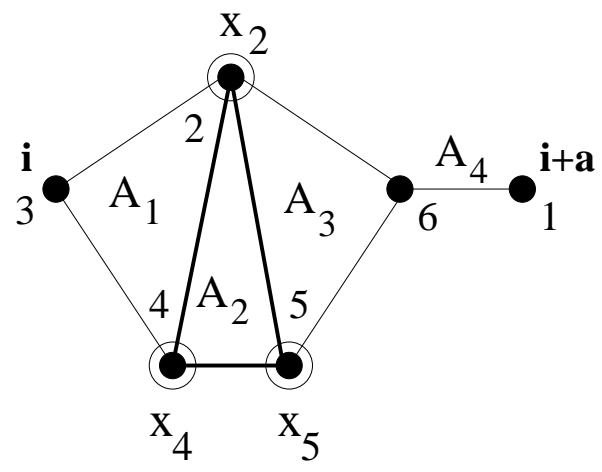

FIG. 2: The block on which the block operator $\hat{B}_{\alpha_{\mathbf{i}}, \sigma_{\mathbf{i}}}^{\dagger}$ is defined at the lattice site $\mathbf{i}$ when connectivity not exists. The block is presented with thick lines, has the form of a triangle, and it contains (see circles) the sites $(2,4,5)$. The coefficients $x_{2}, x_{4}, x_{5}$ are the prefactors [see (11)] of the sites present in the block. 
The relation (17) becomes a supplementary condition leading to the non-connectivity of the localized one-particle states on the flat band. It can be easily checked that when (17) is satisfied, and $x_{2}, x_{4}, x_{5} \neq 0$ holds, we automatically have $x_{\mathbf{j}, 3}=x_{\mathbf{j}, 6}=0$, consequently, connected solutions do not exist.

We further note, that when the condition (17) is satisfied (and given by ([6), the inequality $t_{n}>0$ holds), Eq.(5) which represents the flat band condition, becomes

$$
\epsilon_{0}=2\left(\epsilon_{1}-t_{n}\right)+4 t_{n}, \quad \epsilon_{2}=\frac{t^{2}}{\epsilon_{1}-t_{n}}+\left|t_{c}\right|
$$

In summary, when (6]17,18) are all satisfied, the lowest flat band with one-particle localized states that extends over triangles that do not connect with each other in each unit cell (see Fig.2), the connectivity condition is not satisfied. In this conditions, at half filling lowest flat band, the ground state must be of the form (see (13][15))

$$
\left|\Psi_{g}\right\rangle=\prod_{\mathbf{i}=1}^{N=N_{c}} \hat{B}_{\mathbf{i}, \sigma_{\mathbf{i}}}^{\dagger}|0\rangle,
$$

Here, $\sigma_{\mathbf{i}}$ in each cell is arbitrary, i.e. the ground state is non-magnetic [24].

However, the physical ground state is not of the form (19), i.e., the wave vector (19) does not span the kernel of (8), consequently the uniqueness of (19) as the ground state of the Hamiltonian given in (8) cannot be demonstrated.

The reason why the ground state from (19) is not the true ground sate is as follows. The number $N_{c}$ of linearly independent operators $\hat{B}_{\mathbf{i}, \sigma_{\mathbf{i}}}^{\dagger}$ which were deduced from (12) when the non-connectivity condition (17) holds, are not forming the complete set of solutions of (12). That is, there exists another linearly independent $\hat{B}_{\sigma}^{\dagger}$ operator satisfying (12) when (17) together with ([6) and (18) holds. This operator is extended, and is not related to the states in the lowest flat band. At $t_{c}>0$ it has the form

$$
\begin{array}{r}
\hat{B}_{1, \sigma}^{\dagger}=\sum_{\mathbf{i}}\left[a\left(\hat{c}_{\mathbf{i}+\mathbf{r}_{4}, \sigma}^{\dagger}-\hat{c}_{\mathbf{i}+\mathbf{r}_{5}, \sigma}^{\dagger}\right)\right. \\
\left.+b\left(\hat{c}_{\mathbf{i}+\mathbf{r}_{6}, \sigma}^{\dagger}-\hat{c}_{\mathbf{i}+\mathbf{r}_{1}, \sigma}^{\dagger}\right)\right],
\end{array}
$$

while at $t_{c}<0$ it can be expressed as

$$
\begin{array}{r}
\hat{B}_{2, \sigma}^{\dagger}=\sum_{\mathbf{i}}(-1)^{i}\left[a\left(\hat{c}_{\mathbf{i}+\mathbf{r}_{4}, \sigma}^{\dagger}-\hat{c}_{\mathbf{i}+\mathbf{r}_{5}, \sigma}^{\dagger}\right)\right. \\
\left.+b\left(\hat{c}_{\mathbf{i}+\mathbf{r}_{6}, \sigma}^{\dagger}+\hat{c}_{\mathbf{i}+\mathbf{r}_{1}, \sigma}^{\dagger}\right)\right]
\end{array}
$$


where in the last relation $i=|\mathbf{i}| /|\mathbf{a}|$ is an integer number which represents the length of the vector $\mathbf{i}$ in lattice constant units. In both cases, $b / a=a_{3,5} / a_{3,6}=a_{1,4} / a_{1,3}$ holds for the numerical prefactors in (20)21). We point out that in the process of computing these results, when (6]17][18) holds, $a_{4,1}=a_{4,6} / \operatorname{sign}\left(t_{c}\right)=\sqrt{\left|t_{c}\right|} e^{i \phi_{3}}$ is obtained. For example, a sketch of the $\hat{B}_{1, \sigma}^{\dagger}$ operator is shown in Fig.3.

The study of the eigenvectors of $\tilde{M}$ shows that the one-particle extended states $\left|\phi_{\gamma}\right\rangle=$ $\hat{B}_{\gamma, \sigma}^{\dagger}|0\rangle, \gamma=1,2$ are the $k a=0$ (for $\gamma=1$, note that in this case $t_{c}>0$ ), and $k a=\pi$ (for $\gamma=2$, case in which $t_{c}<0$ ) eigenstates of the dispersive band situated just above the flat band. Since these can be obtained from (12), it points to the fact that these states have energy equal to the flat band. In other terms, for $t_{c}>0$, the dispersive band situated just above the flat band is in contact with the flat band at $\mathbf{k}=0$, while for $t_{c}<0$ at $\mathbf{k a}=\pi$. A sketch at $t_{c}>0$ is shown in Fig.4.

Following these considerations, the physical ground state becomes as follows. We use, for example, the $t_{c}>0$ case (at $t_{c}<0$ the operator $\hat{B}_{1, \sigma}^{\dagger}$ has to be replaced in all equations by $\hat{B}_{2, \sigma}^{\dagger}$ ). In these conditions, at $N=N_{c}+1$ number of electrons we obtain

$$
\left|\Psi_{g}\left(N_{c}+1\right)\right\rangle=\hat{B}_{1, \sigma}^{\dagger} \prod_{\mathbf{i}=1}^{N_{c}} \hat{B}_{\mathbf{i}, \sigma}^{\dagger}|0\rangle
$$

Note that the $\sigma$ index is fixed. This is because $\hat{B}_{1, \sigma}^{\dagger}$, given by the upper dispersive band, enforces the connectivity by being in contact with all operators $\hat{B}_{\mathbf{i}, \sigma}^{\dagger}$. Consequently, the ground state is ferromagnetic, even if the one-particle localized states on the flat band does not satisfy the connectivity condition. Note that at $N_{c}>>1$, the experimental concentration connected to (22) in fact corresponds to the half filled lowest band.

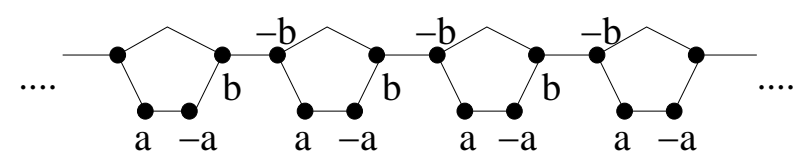

FIG. 3: The infinite block providing the extended operator $\hat{B}_{1, \sigma}^{\dagger}$ being present in all cells at $t_{c}>0$. The black dots denote the sites present in the block, while the coefficients represent the numerical prefactors of the creation operators acting on the given site. 
At $N=N_{c}$ number of electrons we obtain the following structure for $\left|\Psi_{g}\right\rangle$ :

$$
\begin{aligned}
\left|\Psi_{g}\left(N_{c}\right)\right\rangle & =\sum_{i=1}^{N_{c}+1} b_{i}\left[\hat{B}^{\dagger}\left(1, \sigma_{1}\right) B^{\dagger}\left(2, \sigma_{2}\right) \ldots\right. \\
& \times \hat{B}^{\dagger}\left(i-1, \sigma_{i-1}\right) B^{\dagger}\left(i+1, \sigma_{i+1}\right) \ldots \\
& \left.\times \hat{B}^{\dagger}\left(N_{c}, \sigma_{N_{c}}\right) \hat{B}^{\dagger}\left(N_{c}+1, \sigma_{N_{c}+1}\right)\right]|0\rangle
\end{aligned}
$$

where we have denoted in order, the operators $\hat{B}_{1, \sigma}^{\dagger}, \hat{B}_{\mathbf{i}_{1}, \sigma}^{\dagger}, \hat{B}_{\mathbf{i}_{2}, \sigma}^{\dagger}, \ldots \hat{B}_{\mathbf{i}_{c}, \sigma}^{\dagger}$, by the operators present in the set $\mathcal{S}=\left[\hat{B}^{\dagger}(1, \sigma), \hat{B}^{\dagger}(2, \sigma), \ldots, \hat{B}^{\dagger}\left(N_{c}+1, \sigma\right)\right]$. Note that the sum in (23) contains $N_{c}+1$ terms. All terms contain a product of $N_{c}$ operators taken from the set $\mathcal{S}$, such that an arbitrary operator with index $i$ from $\mathcal{S}$ is missing. The numerical prefactor $b_{i}$ holds the index of the missing operator. Note that only the first term from (23) does not satisfy the connectivity condition (hence it has a product of $N_{c}$ operators with arbitrary spin projection). For all other $N_{c}$ terms $i>1$ in (23), containing each a product of $N_{c}$ operators taken from $\mathcal{S}$, one has $\sigma_{1}=\sigma_{2}=\ldots=\sigma_{N_{c}+1}=\sigma$, so the spin projection is fixed. This is enforced by the connectivity condition effective in the $i>1$ terms, introduced by the $\hat{B}_{1, \sigma}^{\dagger}$ operator present in all these contributions. Since only one term from $N_{c}+1$ in (23)

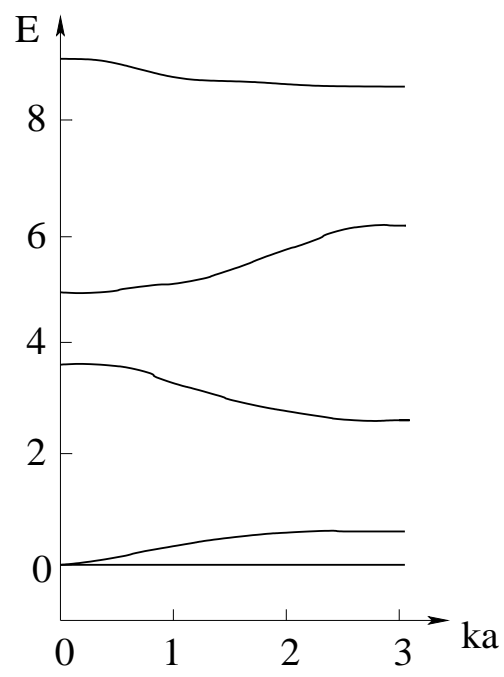

FIG. 4: The band structure of $\hat{H}_{0}$ taken at $t=1, t_{n}=1.2, t_{c}=1.3, \epsilon_{1}=1.5$, when the conditions (6]17[18) are satisfied. Since $t_{c}>0$, the dispersive band placed just above the flat band is in contact with the flat band at $\mathbf{k}=0$. Note that the energy is an even function of $k a$, hence only the $k a>0$ part of the band structure is plotted. 
has arbitrary spin projections, the ground state $\left|\Psi_{g}\left(N_{c}\right)\right\rangle$ represents also a ferromagnetic state at $N_{c}>1$ [25], where $N=N_{c}$ corresponds exactly to the half filled lowest band. (Note that, there are no physical reasons why huge differences should be in a realistic cases between the magnitudes of different $\left|b_{i}\right|$ contributions.)

At the electron number $N<N_{c}$ the ground state becomes of the form

$$
\begin{aligned}
\left|\Psi_{g}\left(N<N_{c}\right)\right\rangle & =\sum_{\mathcal{D}} \alpha_{\left(i_{1}, i_{2}, \ldots, i_{N}\right)}\left[\hat{B}^{\dagger}\left(i_{1}, \sigma_{i_{1}}\right)\right. \\
& \left.\times \hat{B}^{\dagger}\left(i_{2}, \sigma_{i_{2}}\right) \ldots \hat{B}^{\dagger}\left(i_{N}, \sigma_{i_{N}}\right)\right]|0\rangle
\end{aligned}
$$

where $\mathcal{D}=\left\{i_{1}, i_{2}, \ldots, i_{N}\right\}$ is the set of all possible combinations of the integers $\left(i_{1}, i_{2}, \ldots, i_{N}\right)$ labeling the components of $\mathcal{S}$. In this expression the sum has $p_{1}=C_{N_{c}+1}^{N}$ terms [26], and from these only $p_{2}=C_{N_{c}}^{N-1}$ contributions have connectivity conditions, where $p_{2} / p_{1}=N /\left(N_{c}+1\right)$. Hence, with decreasing $N<N_{c}$ and increasing $N_{c}$ the ferromagnetism disappears below the half filled lowest band. The analysis of this crossover exceeds the frame of the present Letter and will be discussed elsewhere.

We mention that the ground states from (22,23,24) being constructed at the mentioned $N$ with the complete set of solutions of (12) are unique since span the kernel of (8). The results remain valid even if only sites $\mathbf{i}+\mathbf{r}_{4}$ or $\mathbf{i}+\mathbf{r}_{5}$ are only interacting (hence $80 \%$ of sites are non-interacting in the system). We further note that stability studies made for flat band ferromagnetism before not include the here presented case [27].

In conclusions, in a system in which there is a lowest bare flat band of one-particle localized states which do not satisfy the connectivity condition, the flat band ferromegnetism does not work and the ground state of the half filled lowest band is not a ferromagnet. Contrary to this, we rigorously proved that, in some circumstances ferromagnetism is still possible. The reason for this is that the dispersive band which appears just above the lowest flat band can be forced to be in contact with the lowest flat band. This contact point represents a particular extended one particle state which belongs to the dispersive band, but which has the energy of the one particle states from the flat band. This state being extended, will introduce the connectivity condition, enforcing a ferromagnetic state. We showed that this phenomenon exists in a class of pentagon chains in which the conditions leading to the lowest flat band containing the non-connected localized one-particle states, automatically leads to one contact point with the dispersive band situated just above the flat band. 
We underline that this is not a rare effect. Indeed, for a kinetic Hamiltonian $\hat{H}_{0}$ with several hopping matrix elements $t_{\nu}$ and one particle potentials $\epsilon_{\nu}$, the bare band energies $\epsilon$ can be obtained usually from a relation of the type $A\left(\left\{\epsilon, t_{\nu}, \epsilon_{\nu}\right\}\right)+B\left(\left\{\epsilon, t_{\nu}, \epsilon_{\nu}\right\}\right) \cos \mathbf{k a}=0$ [28], and the minimum distance (i.e. gap) between the lowest and the second band can be denoted by $\Delta\left(\left\{t_{\nu}, \epsilon_{\nu}\right\}\right)$. Choosing zero energy scale, the presence of a bare flat band means $A\left(\left\{0, t_{\nu}, \epsilon_{\nu}\right\}\right)=B\left(\left\{0, t_{\nu}, \epsilon_{\nu}\right\}\right)=0$, the placement of the flat band in the lowest position

representing a supplementary condition $F\left(\left\{t_{\nu}, \epsilon_{\nu}\right\}\right)=0$. Furthermore, the presence of a contact point between the lowest flat band and the dispersive band situated just above can be simply given by $\Delta\left(\left\{t_{\nu}, \epsilon_{\nu}\right\}\right)=0$. These four equations (i.e. $A=0, B=0, F=0, \Delta=0$ ) always provide solutions where the number of Hamiltonian parameters in $\hat{H}_{0}$ - as in realistic cases (e.g., in the studied poly(3-alkylthiophene) polymer pentagon chain case is seven) - is high.

\section{ACKNOWLEDGMENTS}

For M. Gulácsi this research was realized in the frames of TAMOP 4.2.4. A/2-11-1-20120001 "National Excellence Program - Elaborating and operating an inland student and researcher personal support system". The project was subsidized by the European Union and co-financed by the European Social Fund. Z. Gulácsi kindly acknowledges financial support provided by the Alexander von Humboldt Foundation, OTKA-K-100288 (Hungarian Research Funds for Basic Research) and TAMOP 4.2.2/A-11/1/KONV-2012-0036 (co-financed by EU and European Social Fund).

[1] Y. Zhang, Y. W. Tan, H. L. Stormer, and P. Kim, Nature 438, 201 (2005).

[2] M. Z. Hasan and C. L. Kane, Rev. Mod. Phys. 82, 3045 (2010).

[3] R. Takahashi and S. Murakami, Phys. Rev. B. 88, 235303 (2013).

[4] G. Möller and N. R. Cooper, Phys. Rev. Lett. 108, 045306 (2012).

[5] O. Derzhko, J. Richter, A. Honecker, and R. Moessner, Phys. Rev. B. 81, 014421 (2010).

[6] Z. Gulácsi, Phys. Rev. B. 77, 245113 (2008).

[7] A. Mielke and H. Tasaki, Commun. Math. Phys. 158, 341 (1993). 
[8] A. Mielke, J. Phys. A24, L73 (1991); ibid A24, 3311 (1991); ibid. A25, 4335 (1992).

[9] O. Derzhko and J. Richter, Phys. Rev. B. 90, 045152, (2014); R. Trencsényi and Z. Gulácsi, Eur. Phys. Jour. B. 75, 511, (2010).

[10] Z. Gulácsi, A. Kampf, D. Vollhardt, Phys. Rev. Lett. 99, 026404 (2007); Z. Gulácsi, A. Kampf, D. Vollhardt, Progr. Theor. Phys. Suppl. 176, 1 (2008).

[11] Z. Gulácsi, A. Kampf, D. Vollhardt, Phys. Rev. Lett. 105, 266403 (2010).

[12] Z. Gulácsi, Int. Jour. Mod. Phys. B. 27, 1330009 (2013).

[13] Z. Gulácsi, Eur. Phys. Jour. B87, 143 (2014).

[14] M. Gulácsi, G. Kovács, and Z. Gulácsi, Phil. Mag. Lett. 94, 269 (2014).

[15] I. Orlik, Z. Gulácsi, Phil. Mag. Lett. 78, 177 (1998); Z. Gulácsi, I. Orlik, Jour. of Phys. A. 34, L359 (2001).

[16] P. Gurin and Z. Gulácsi, Phys. Rev. B. 64, 045118 (2001); Z. Gulácsi, Eur. Phys. Jour. B 30, 295 (2002); Phys. Rev. B. 66, 165109 (2002).

[17] Z. Gulácsi and D. Vollhardt, Phys. Rev. Lett. 91, 186401 (2003); Z. Gulácsi and D. Vollhardt, Phys. Rev. B. 72, 075130 (2005).

[18] Z. Gulácsi, Phys. Rev. B. 69, 054204 (2004).

[19] Z. Gulácsi, M. Gulácsi, Phys. Rev. B. 73, 014524 (2006).

[20] A. J. Heeger, S. Kivelson, J. R. Schrieffer, and W. P. Su, Rev. Mod. Phys. 60, 781 (1988).

[21] A. S. Dhot, G. M. Wang, D. Moses, and A. J. Heeger, Phys. Rev. Lett. 96, 246403 (2006); C. Tanase, E. J. Meijer, P. W. M. Blom, and D. M. de Leeuw, Phys. Rev. Lett.91, 216601 (2003); A. C. R. Grayson, I. S. Choi, B. M. Tayler, P. P. Wang, H. Brem, M. J. Cima, and R. Langer, Nature Mater. 2, 767 (2003); R. Arita, Y. Suwa, K. Kuroki and H. Aoki, Phys. Rev. Lett. 88, 127202 (2002).

[22] R. McNeill, R. Siudak, J. H. Wardlaw, and D. E. Weiss, Austr. Jour. Chem. 16, 1056 (1963); J. W. van der Horst, P. A. Bobbert, and M. A. J. Michels, Phys. Rev. Lett. 83, 4413 (1999).

[23] H. Sirringhaus, N. Tessler and R. H. Friend, Science 280, 1741 (1998).

[24] Note that for $N<N_{c}$ the ground state (19) becomes a sum of the form $\sum_{\mathbf{i}_{1}, \ldots, \mathbf{i}_{N}} \alpha_{\mathbf{i}_{1}, \ldots, \mathbf{i}_{N}} \prod_{\mathbf{i}=1}^{N} \hat{B}_{\mathbf{i}, \sigma_{\mathbf{i}}}^{\dagger}|0\rangle$, where the product is made over all possible $\left(\mathbf{i}_{1}, \ldots, \mathbf{i}_{N}\right)$ sets. Because the connectivity is missing, this state is paramagnetic.

[25] i.e. $\left\langle\Psi_{g}\left(N_{c}\right)\left|\hat{S}^{2}\right| \Psi_{g}\left(N_{c}\right)\right\rangle \neq 0$ holds.

[26] Here $C_{n_{2}}^{n_{1}}$ means the number of combinations of $n_{2}$ objects on $n_{1}<n_{2}$ places. 
[27] e.g. in A. Mielke, J. of Phys. A32, 8411 (1999) because of eq.(10) and $S=S_{z}=N_{d} / 2$, the results are valid only for $N_{d}=N_{\text {sites }}$, while in our case, the degree of degeneracy $N_{d}=N_{c}+1$, while the total number of sites is $N_{\text {sites }}=5 N_{c}$.

[28] In higher dimensions, the bare band structure can emerge in the form $\sum_{n=0}^{m} A_{n} \cos ^{n} \mathbf{k a}=0$, where $m$ is finite. In this case, the presence of a flat band can be given as $A_{n}=0$ for all $n$. 